\title{
ATTO: Wireless Networking at Fiber Speed (invited)
}

\author{
Guy Torfs, Member, IEEE, Haolin Li, Student Member, IEEE, Sam Agneessens, Member, IEEE, \\ Johan Bauwelinck, Member, IEEE, Laurens Breyne, Olivier Caytan, Student Member, IEEE, Wout \\ Joseph, Senior Member, IEEE, Sam Lemey, Member, IEEE, Hendrik Rogier, Senior Member, IEEE, \\ Arno Thielens, Member, IEEE, Dries Vande Ginste, Senior Member, IEEE, Joris Van Kerrebrouck, \\ Student Member, Gunter Vermeeren, Member, IEEE, Xin Yin, Member, IEEE, Piet Demeester, \\ Fellow, IEEE
}

\begin{abstract}
ATTO targets wireless networking at fiber speed: $100 \mathrm{Gbps} / \mathrm{m}^{2}$ with latencies smaller than $10 \mu \mathrm{s}$. To provide this tremendous wireless capacity, ultra-small floor-integrated cells are proposed. In this way, short-reach communication can be established, reducing the effect of interference and providing full frequency reuse in the wireless domain. RF-over-fiber, coherent communication and a dedicated 2D PON structure support the interconnection and selection of the cells, and minimize the required transceiver electronics. To evaluate the feasibility of the proposed architecture, key principles are validated at lower frequency bands. Two main building blocks are addressed in this paper: A fully passive opto-antenna, to prove that a passive remote antenna head can be realized owing to the short transmission distances. Furthermore, a low-cost RF-over-fiber system is demonstrated: sigma-delta modulation drives non-linear optical modulators, such as electro-absorption modulators and verticalcavity surface-emitting lasers using a digital transmitter while remaining compatible with the passive opto-antenna. Finally, two important properties of the ATTO-floor are evaluated. The exposure of a human body model to RF fields by the antenna floor. Measurements ensure a 200-fold margin with respect to the ICNIRP basic restriction. To guarantee that multiple devices can communicate with the ATTO-floor simultaneously, the interference between cells spaced $300 \mathrm{~mm}$ apart was evaluated.
\end{abstract}

Index Terms-100 Gbps Wireless communication, RF-overfiber, Matched Photodiode, Opto-Antenna, Sigma-delta modulation, Exposure, Interference

\section{INTRODUCTION}

$\mathrm{T}$ HE emergence of the fifth generation $(5 \mathrm{G})$ cellular network brings along new challenges in the wireless access domain[1]. A massive growth of devices and connected things is foreseen and the mobile data traffic is skyrocketing with the arrival of new technologies such as cloud computing, ultra-high definition video streaming and virtual reality.

This work was supported in part by the ERC grant 695495 "ATTO: A new concept for ultra-high capacity wireless networks", the European Commission via H2020 5G-Phos (grant 761989) and the Research Foundation Flanders.

G. Torfs, H. Li, J. Bauwelinck, L. Breyne, O. Caytan, S. Lemey, H. Rogier, D. Vande Ginste, J. Van Kerrebrouck, X. Yin and P. Demeester are with the Department of Information Technology, IDLab, Ghent University - imec, Ghent, Belgium (email: guy.torfs@ugent.be).
To anticipate other future applications, such as distributed robotic systems in a factory-of-the-future environment, wireless access to high-performance computing resources is required. It is envisioned that a futuristic factory will be populated by numerous autonomous robots. To perform the most difficult tasks in a versatile manner, these robots require a high degree of intelligence. Moreover, they should be able to react very fast to changing conditions (including interactions with humans) or operate within time-critical control loops. This human-like intelligence requires a tremendous amount of computing power. In the future, robots will process data at tens of teraflops. Yet, by combining their computing power with that of the other robots sharing the same area and/or with local computing power in their immediate environment, they become an integral part of a petaflops computer. However, the demands in terms of wireless communication greatly surpass the currently proposed 5G specifications. The required interconnection speeds (dedicated bitrates up to $100 \mathrm{Gbps}$ ), extremely low latencies (down to $10 \mu \mathrm{s}$ ) - defined as the time between IP traffic entering and exiting the network -, high densities (e.g. 100 robots on $250 \mathrm{~m}^{2}$ ) and high reliability are today only encountered in "wired", datacenter-oriented, connection systems using for example cabled InfiniBand technology. To satisfy these requirements, we propose a novel wireless access architecture (ATTO), which provides bitrates of $100 \mathrm{Gbps}$ with latencies below $10 \mu \mathrm{s}$, data densities of 100 $\mathrm{Gbps} / \mathrm{m}^{2}$ and this with very high reliability (for mobility of ground objects moving at less than $30 \mathrm{~km} / \mathrm{h}$, such as robots or humans). The system concept consists of a collection of very small cells (with a typical size of $15 \times 15 \mathrm{~cm}^{2}$ ), which are integrated into the floor. The floor integrated antenna of the cell will be able to set up a dedicated 100 Gbps communication stream to a floor-facing antenna of the mobile object positioned on top of the cell. A $3 \times 3$ line-of-sight MIMO channel using a

W. Joseph, A. Thielens and G. Vermeeren are with The Department of Information Technology, Ghent University - imec, Ghent, Belgium

Sam Agneessens is with the Department of Information Technology, IDLab, Ghent University - imec and the Centre for Microsystems Technology (CMST), Ghent University - imec, Ghent, Belgium.

Copyright (c) 2015 IEEE. Personal use of this material is permitted. However, permission to use this material for any other purposes must be obtained from the IEEE by sending a request to pubs-permissions@ieee.org. 


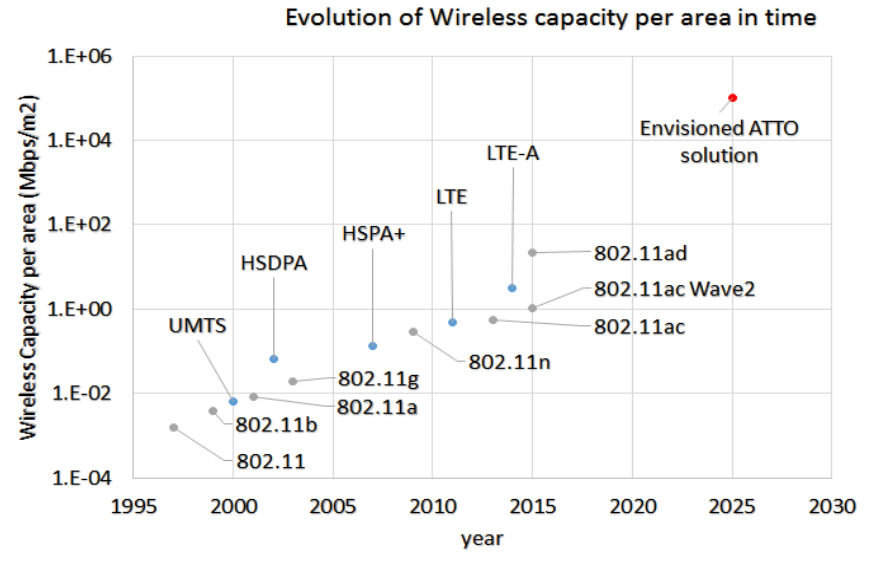

Fig. 1. Bitrate densities of today's standardized wireless RF technologies.

quadrature-amplitude-modulated signal with a constellation of 64 symbols (64-QAM) in the $60 \mathrm{GHz}$ band will be used. Compared to the proposed $5 \mathrm{G}$ specifications, this represents an increase by 1000 in bitrate/area, a reduction by 100 in latency and an increase by 10 in bitrate.

This paper is an invited extension of [2] and continues with an overview of the performance of wireless standards, especially focusing on achievable data rate per area. Next, in section III, our ATTO concept is introduced. The optical antenna interconnection system is discussed in more detail in section IV. Section V focusses on some recent subsystem developments and section VI discusses the interference level and exposure associated with the proposed system. The paper ends with a conclusion in section VII.

\section{EVOLUtion OF WIRELESS PERFORMANCE}

It is clear that currently no wireless technology is capable of delivering the required performance: $100 \mathrm{Gbps} / \mathrm{m}^{2}$ and latencies of less than $10 \mu \mathrm{s}$. The bitrate densities per area of today's major standardized wireless RF technologies [3], [4] are depicted in Fig. 1.

The technologies considered include IEEE 802.11 ('99, a, b, $\mathrm{g}, \mathrm{n}, \mathrm{ac}, \mathrm{ad})$ using their typical transmission range, and 3GPP
(UMTS, HSDPA, HSPA+, LTE, LTE-Advanced), using femto cells with a radius of 10 meters. For example, the $20 \mathrm{Mbps} / \mathrm{m}^{2}$ point corresponds to today's $60 \mathrm{GHz}$ IEEE 802.11ad technology with a typical indoor range of $10 \mathrm{~m}$. The envisioned goal, being a bitrate density of $100 \mathrm{Gbps} / \mathrm{m}^{2}$, as indicated in red, is targeted for the year 2025 .

None of the mentioned standards were specifically developed to increase the capacity per unit of area. To maximize the available data rate, the total available spectrum should be exploited in an optimal manner. Instantaneously minimizing the latency demands for a dedicated cell or access point per user. Hence, with the aim of realizing a wireless capacity density of $100 \mathrm{Gbps} / \mathrm{m}^{2}$, ultra-small cells become critically important, and to be consistent with the existing scaling paradigm, these small cells are denoted ATTO-cells.

\section{ATTO-CELLS}

Currently, there is no technology meeting all these stringent requirements. A very large bandwidth is needed to realize the required 100 Gbps throughput and, as a result, only millimeterwave frequencies can be considered feasible candidates. To increase the available capacity per square meter, the cell size needs to be reduced as much as possible, together with the interference between adjacent active cells.

Therefore, we introduce a radically new approach: the deployment of very small antenna cells (ATTO-cells) integrated in floors.

An overview of this system is given in Fig. 2. The depicted ATTO-macroblock, comprising multiple ATTO-cells, has a dedicated gateway, where most of the electronics are aggregated and which provides access to the Internet or other ATTO-macroblocks. The gateway connects to the different ATTO-cells using a RF-over-fiber [5] passive optical network (PON) connection.

\section{ANTENNA INTERCONNECTION SYSTEM}

Due to the high number of cells, it is no longer economically feasible to integrate a complete transceiver at every ATTO-cell. Hence, only a limited number of transceivers will be

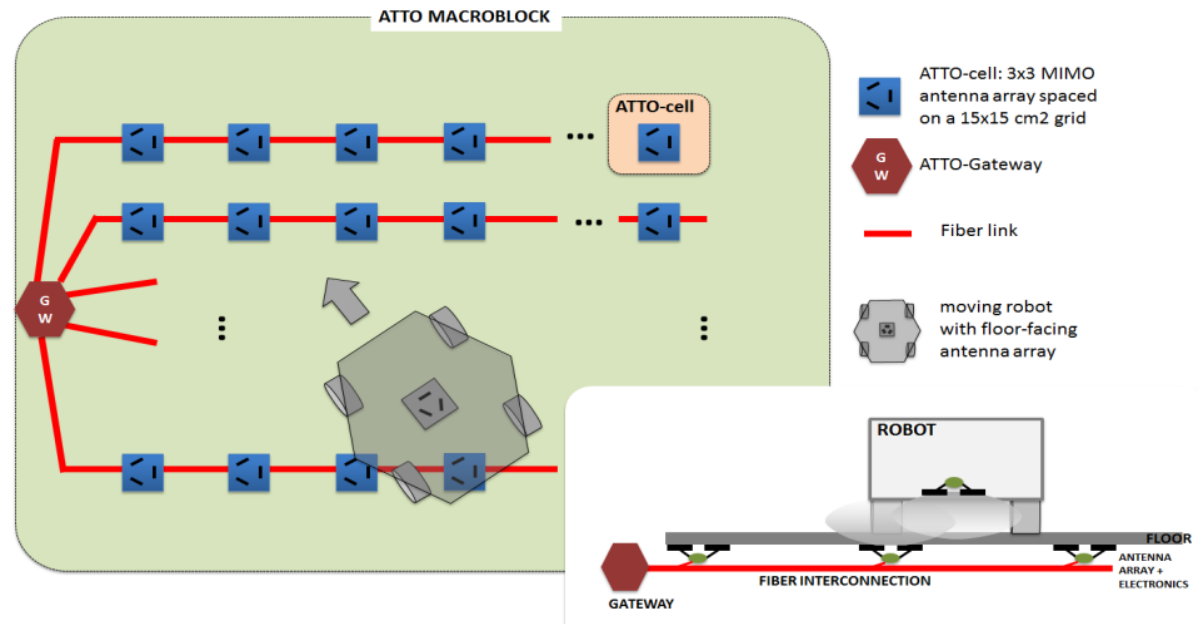

Fig. 2. ATTO-concept: highly intelligent robots on a sea of antennas. 


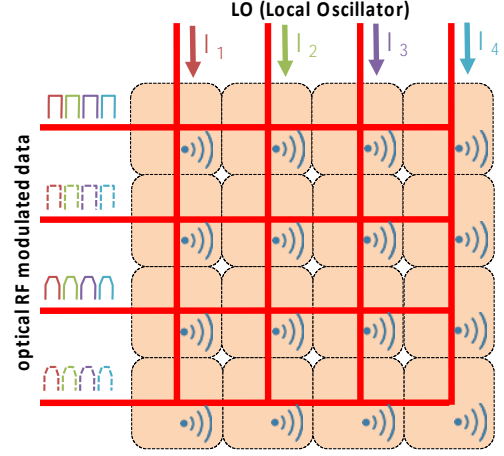

(a)

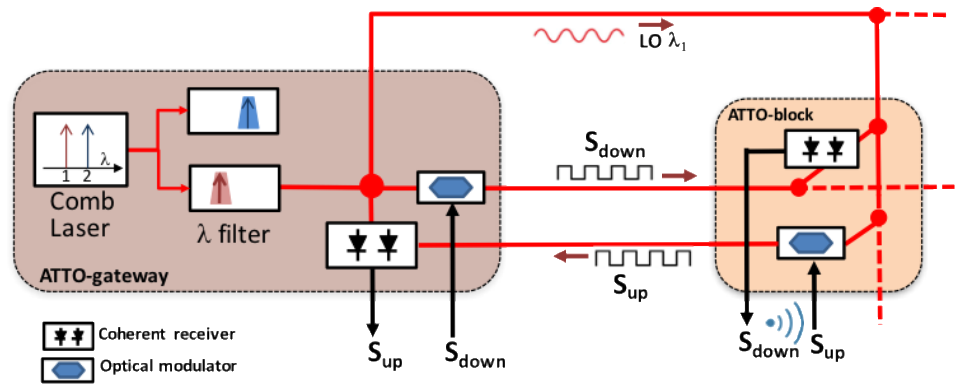

(b)

Fig. 3: (a) Basic concept of the optical interconnect architecture using "horizontal data" PONs and "vertical selection" PONs, (b) Principle of the use of a single laser to produce both the data carrier and LO in upstream and downstream, drastically reducing complexity in the ATTO-cell

provisioned and these will be integrated into a common ATTOgateway. In this way, the cost of an ATTO-cell can be kept low. The total amount is part of the planning process and relates to the number of mobile nodes foreseen in the area covered. In fact, the number of transceivers used simultaneously corresponds to the number of active ATTO-cells, which is much smaller than the total amount of ATTO-cells.

Another major challenge is the development of an optical interconnection network that efficiently connects the ATTOcells with the ATTO-gateway using a dedicated path (using wavelength division multiplexing over different fibers). It is important to recall that the primary service offered to each user is access to a full $100 \mathrm{Gbps}$ stream that is not shared amongst other users. The different transceivers need to be dynamically connected to the different ATTO-cells, requiring a flexible, high-bandwidth reconfigurable optical-fiber interconnection network. To enable flexible addressing of the different ATTOcells, a parallel coherent PON is proposed in Fig. 3a.

The usage of RF-over-fiber limits the electronics required at the ATTO-cells to amplifiers and opto-electrical components. Due to the high number of ATTO-cells, it is not cost-effective to deploy a dedicated fiber for each cell, while the gateway would require the same number of optical transceivers. To keep the number of fibers low and to limit the split factor, we propose an interconnection network consisting of "horizontal data" and

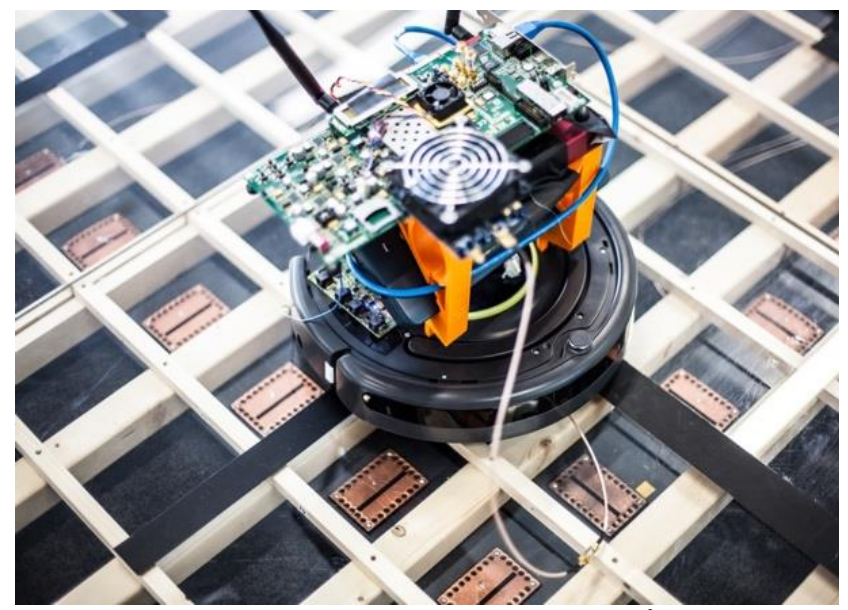

Fig. 4: ATTO proof-of-concept demonstrator: $3 \mathrm{Gbps} / \mathrm{m}^{2}$ (unidirectional), $3.5 \mathrm{GHz}$ band. "vertical selection" PONs, as in Fig. 3a. The downstream data streams for a particular row are multiplexed on different wavelengths ( $\mathrm{N}$ in total) and the combined signal propagates from left to right. The wavelength associated with a particular antenna is selected automatically in a coherent receiver by the local oscillator (LO) wavelength provided via the vertical PONs. Both the modulation wavelength and the $\mathrm{LO}$ wavelength are generated by the same source (see Fig. 3b).

The upstream signals from right to left can reuse the same LO carrier using external optical modulators, but they travel on a separate horizontal PON to avoid crosstalk. Furthermore, all the different wavelengths applied in the architecture originate from one comb laser in the ATTO-gateway. As a result, frequency drift is mitigated and no expensive temperature stabilization is required in the ATTO-cells. Moreover, because the local oscillator used to generate the signal is reused at the receive side, the specifications on the laser linewidth are relaxed. The coherence time of the laser should be longer than the difference in traveling time of the data signal and local oscillator signal. This difference can be made small through careful floor planning of the ATTO-floor.

The use of the LO wavelength to select the right ATTO-cell results in a non-blocking flexible architecture. The use of a single laser that acts as signal carrier and LO in both upstream and downstream direction yields a very efficient reuse of components at the ATTO-gateway, and a strongly reduced complexity at the ATTO-cells. Moreover, if a sufficiently strong LO is applied, the coherent detection introduces gain in the optical-electrical conversion which can be used to compensate for the splitting losses in the PON architecture.

\section{SUBSYSTEM DEVELOPMENT}

A prototype of the wireless part is illustrated in Fig. 4: the robot, the $15 \times 15 \mathrm{~cm} 2$ ATTO-cells and the slotted antennas integrated in the floor. To realize an economically feasible solution, very-low-cost and low-power remote radio heads (RRHs) are essential components to meet the requirements of the proposed ATTO system. The integration of a massive amount of antennas into the floor calls for a nearly passive approach to integrate the required infrastructure into the environment. To eventually arrive at such a solution, we now 


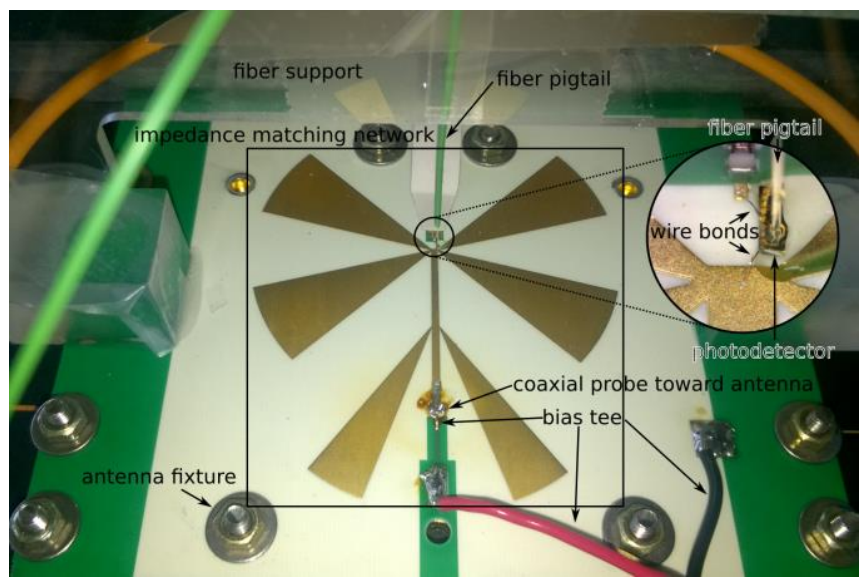

(a)

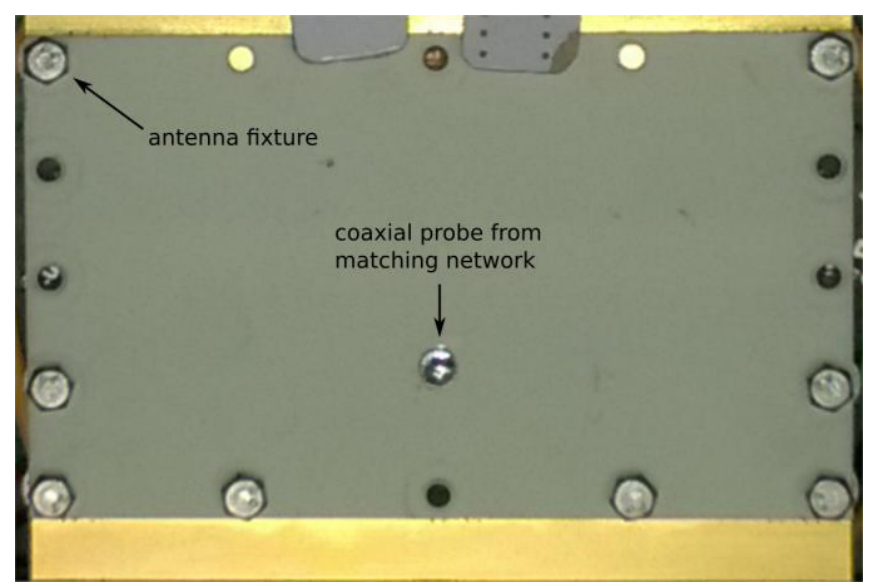

(b)

Fig. 5: Prototype of the remote radio head (RHH) fully passive downstream subsystem. (a) Back-side depicting the fiber, photodetector, matching network and bias tee (short-circuited in this experiment). (b) Front-side depicting the air-filled substrate-integrated-waveguide (AFSIW) cavity-backed slot antenna.

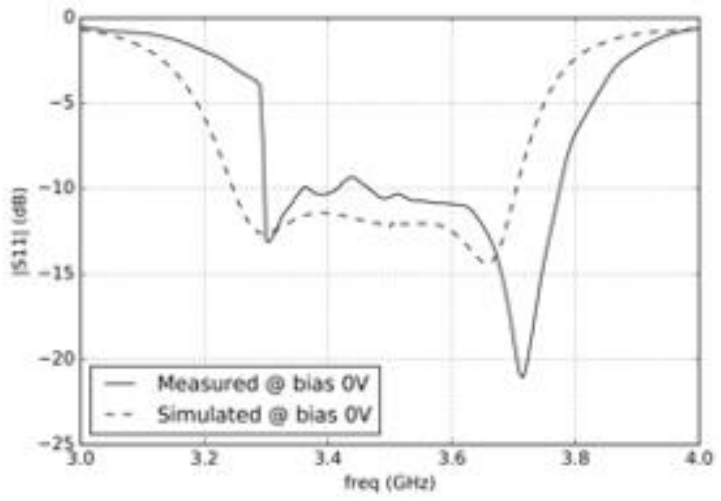

Fig. 6: Simulated and measured reflection coefficient of the matched photodiode.

discuss two intermediate developments operating at lower frequencies, yet designed to validate the underlying principles. First, we describe a fully passive opto-antenna, which solely consists of a photodiode with zero bias, matched to a dedicated substrate-integrated-waveguide (SIW) antenna. Second, we outline sigma-delta modulation, which can be used in a lowcost all-digital transmitter.

\section{A. Fully passive SIW-based opto-antenna}

The RF-over-fiber scheme already reduces the complexity of the hardware required at each ATTO-cell significantly. It only encompasses opto-electrical conversion components, electrical amplifiers, and antennas. In the downstream direction, this becomes a photodetector with transimpedance amplifier, a power amplifier and a transmit antenna.

However, because of the small cell size and the correspondingly short propagation distance between the ATTO-cell and the mobile user, the link budget is characterized by a very small propagation loss. Therefore, the transmitted RF power per cell can be kept very low. It turns out that, in this situation, the transimpedance and power amplifier can be replaced by only a passive impedance matching network, while still radiating adequate RF power. Based on an accurate characterization of the photodetector, a passive matching network can be designed to transform the impedance of the transmit antenna into the complex conjugate of the photodetector impedance over the specified frequency bandwidth, thereby achieving optimal power extraction over the complete frequency band [6]. The simulated and measured reflection coefficient of the matched photodiode is shown in Fig. 6. Furthermore, the modest RF power allows the photodetector to operate at zero bias without introducing significant non-linear distortion. The resulting zero-bias passive opto-antenna enables the deployment of RRHs that remain fully passive in the downstream path, and whose radiated power originates entirely from the optical power incident on the photodetector.

We have validated these techniques by developing a prototype at $3.50 \mathrm{GHz}$ (FCC's innovation band) of the described downstream RRH subsystem, as shown in Fig. 5. The RF signal is supplied to the RRH by a single multi-mode optical fiber, which is pigtailed onto the photodetector with an indexmatching epoxy and mechanically supported by a Plexiglass structure. This prototype uses a 25 Gbps PIN photodetector, with a peak responsivity near $0.85 \mu \mathrm{m}$. However, the technique can be readily extended towards other RF frequency bands and wavelengths. The photodetector contacts are connected to the impedance matching network on the printed circuit board

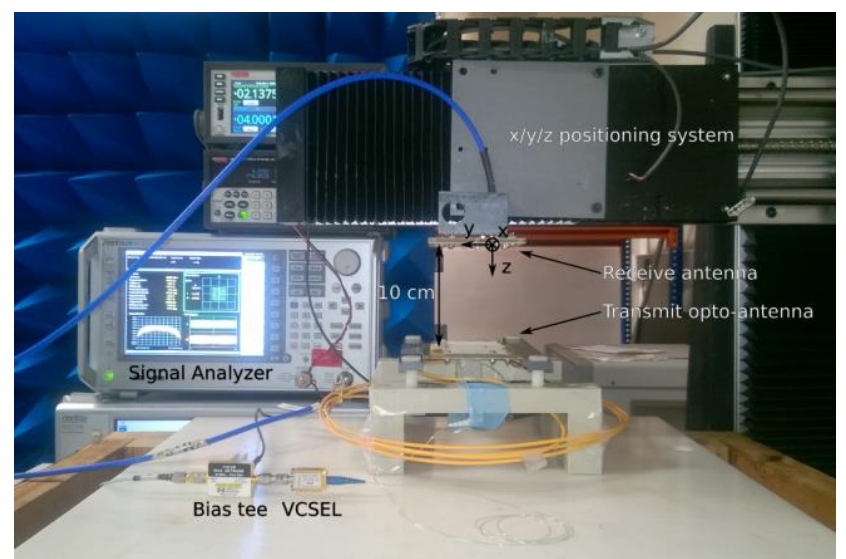

Fig. 7: Downstream communication link setup with the remote radio head $(\mathrm{RRH})$ prototype in a realistic propagation environment. 


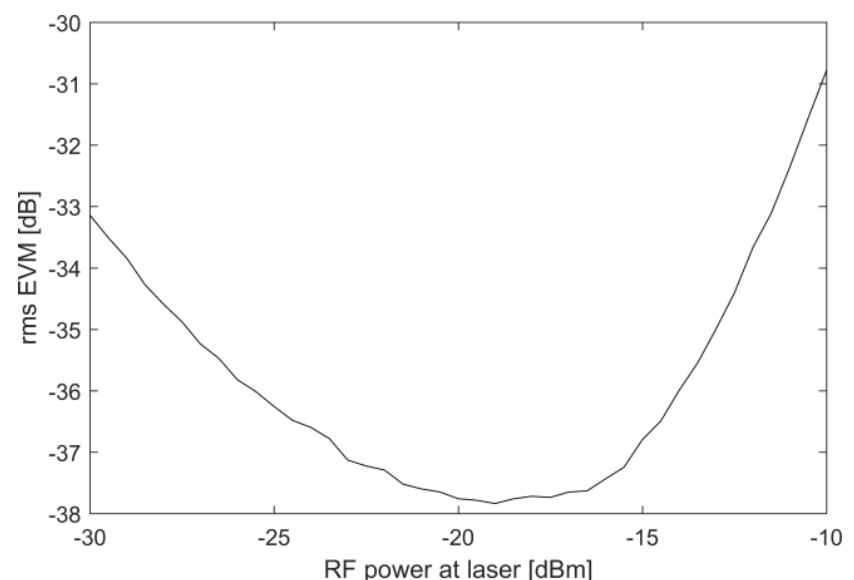

Fig. 8: Measured rms EVM vs. RF power applied at the laser for a 64QAM signal.

(PCB) through wire bonds. The on-board bias tee is shortcircuited, as the photodetector operates at zero bias. The matching network is designed for optimal power transfer from the photodetector to a planar air-filled substrate-integratedwaveguide (AFSIW) antenna [7], [8], [9] with $50 \Omega$ radiation impedance around $3.5 \mathrm{GHz}$. The probe-fed antenna is compactly mounted onto the back-plane of the PCB which contains the matching network. The adopted antenna topology is inherently broadband, and can therefore be optimized to exhibit a low reflection w.r.t. $50 \Omega$ across a wide frequency band and thanks to the air substrate, a very high radiation efficiency is obtained which further increases the impedance bandwidth.

The performance is verified by employing the $\mathrm{RRH}$ prototype as the transmit antenna in a unidirectional data link set up in a realistic propagation environment, as depicted in Fig. 7. An Anritsu MG3710A Vector Signal Generator generates a 64-QAM with a symbol rate of $80 \mathrm{MBd}$ and a carrier frequency of $3.50 \mathrm{GHz}$. A VI Systems V50-850M Multi-Mode FiberCoupled vertical-cavity surface-emitting laser (VCSEL) is biased with a current of $4 \mathrm{~mA}$, and modulated with the 64-QAM signal. Its optical output is connected to the RRH prototype, which is aligned for maximum radiation along the $-\mathrm{z}$ direction. Another AFSIW antenna is attached to an $x / y / z$ positioning system and is oriented for optimal reception along the $+\mathrm{Z}$ direction, with its polarization aligned with that of the RRH. The distance between both antennas along the $\mathrm{z}$-axis, equals 10 $\mathrm{cm}$. The received signal is amplified by a Mini-Circuits ZX6083LN-S+ Low Noise Amplifier (powered by a 5 V DC source) and received by an Anritsu MS2692A Signal Analyzer, which measures the received signal power and demodulates the 64QAM signal to calculate the root-mean-square error-vectormagnitude (rms EVM). Both transmitter and receiver use a root-raised-cosine filter with roll-off factor 0.50 .

In a first experiment, the phase centers of transmit and receive antenna are perfectly aligned such that their separation is only $10 \mathrm{~cm}$. The power of the 64-QAM signal applied at the VCSEL is optimized to minimize the EVM of the link. Indeed, when the signal power is too low, the EVM will be limited by the signal-to-noise ratio (SNR), while the EVM will be limited

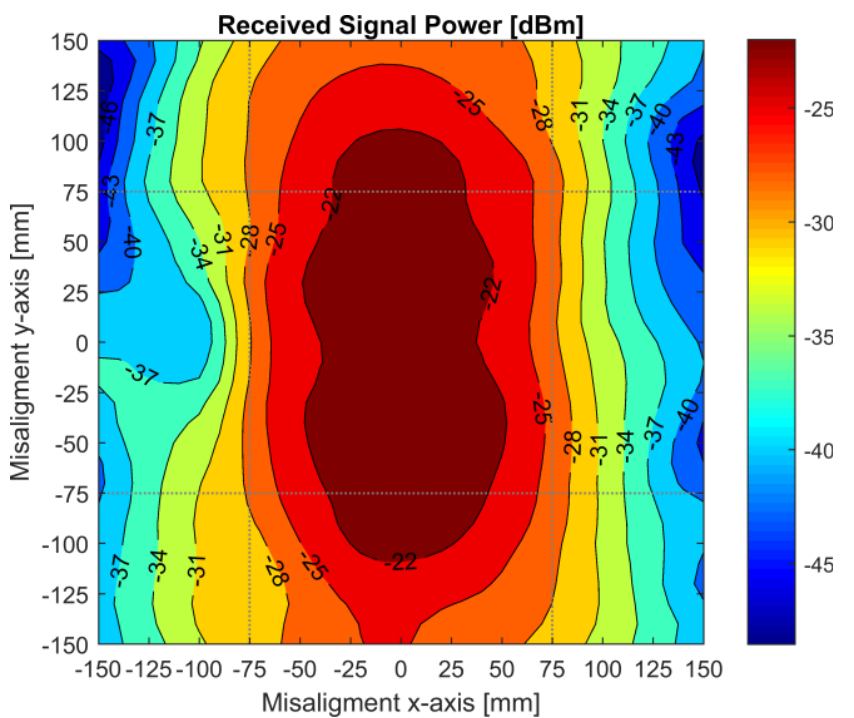

Fig. 9: Measured received signal power vs. receive antenna misalignment. The boundaries of one ATTO-cell are indicated by the dotted lines.

by the non-linear distortion introduced by the VCSEL and the photodetector with zero bias when the signal power is too high. Fig. 8 shows a measurement of the rms EVM as a function of RF power, which indicates that the optimum RF power is -18 $\mathrm{dBm}$. This signal power is applied for all subsequent measurements.

Subsequently, the $\mathrm{x} / \mathrm{y} / \mathrm{z}$ positioning system is used to evaluate signal power and EVM on a dense grid of positions in the XY plane, covering an area of four ATTO-cells. The measured signal power is presented in Fig. 9. Within the entire central ATTO-cell, indicated by the grey dotted lines, the link performed excellently, with an rms EVM below -34dB.

\section{B. All-digital opto-transmitter}

To interconnect all ATTO-cells, a PON fiber back-bone is proposed. In essence, there are two ways of transmitting a radio signal over fiber. Both techniques are depicted in Fig. 10. The most straightforward approach is Analog Radio-over-Fiber (ARoF). Here, a digitized signal is transformed to the analog domain using a digital-to-analog converter (DAC) and, if needed, upconverted to an RF frequency. It is as such transmitted through an optical link. This is definitely the most spectral-efficient solution and it is fully compatible with the proposed opto-antenna solution. However, this approach is susceptible to analog impairments and it requires a highly linear

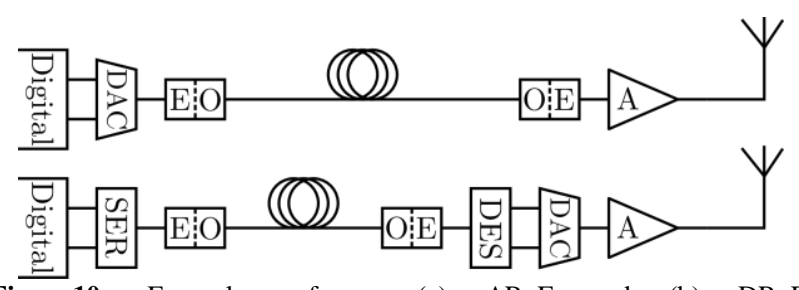

Fig. 10: Examples of an (a) ARoF and (b) DRoF. E-O: Electrical-to-Optical; O-E: Optical-to-Electrical; A: Amplifier; SER: Serializer; DES: Deserializer. 


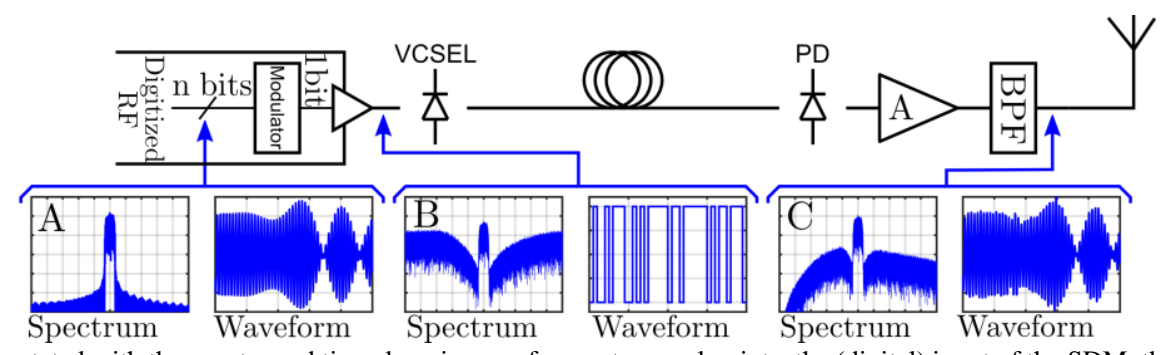

Fig. 11: The SDoF link annotated with the spectra and time domain waveforms at several points: the (digital) input of the SDM, the (electrical) output of the SDM and the output of the opto-antenna.

and high bandwidth opto-electrical (O/E) modulator. A second approach is Digital Radio-over-Fiber. Here, digital I/Q samples are serialized and transmitted using a standardized protocol such as CPRI [10]. However, at the receive side, the data should be converted to an analog signal before it can be transmitted by the antenna. It is clear that the medium conversions between optical, digital and RF at the RRH will prohibit a low-cost and a low power solution.

Alternatively, we propose to transmit a sigma-delta modulated signal (sigma-delta-over-fiber, SDoF [11]) taking the advantage of both digital transmission while keeping a low complexity RRH (Fig. 11). A digital RF signal (inset A) is generated and then converted to a one-bit signal using a parallelized sigma-delta modulator (SDM). This modulator oversamples the input data and quantizes it in such a way that the quantization noise is shaped outside the frequency band of interest. The resulting one-bit (digital) signal (inset B) can then be transmitted using similar components as used for DRoF. The two main advantages of this approach are that any nonlinearities of $\mathrm{E} / \mathrm{O}$ modulators are mitigated and that the spectrum of this signal already contains the required RF signal. Hence, when receiving this signal at the RRH using the bandlimited opto-antenna, the quantization noise is filtered out and the RF signal of interest is recovered without any additional signal processing (inset $\mathrm{C}$ ).

To investigate this approach, both ARoF and SDoF are compared. An analog 16-QAM signal modulated at $1 \mathrm{GHz}$ carrier frequency with different baud rates was transmitted using a directly modulated $850 \mathrm{~nm}$ multimode VCSEL, being the same laser as used in the opto-antenna measurements, and received using a resonant transimpedance amplifier (TIA) [12]. A similar signal was also sigma-delta modulated with a sampling rate of $10 \mathrm{GSps}$ and transmitted using the same VCSEL, only this time the VCSEL is driven using a non-returnto-zero on-off-keying signal. At the receive side, the same resonant TIA is used.

The performance is visualized in Fig. 12. Here, the EVM of the received RF signal is shown for different VCSEL bias currents when it is modulated with the analog or with the sigmadelta signal and this for different baud rates. An EVM improvement between 2.4 and $7.1 \mathrm{~dB}$ is observed when comparing SDoF with ARoF.

To extend this technique to higher carrier frequencies and symbol rates, two adaptations are proposed. Higher carrier frequencies are realized by splitting modulation and carrier frequency. A single wave length is modulated coherently, for

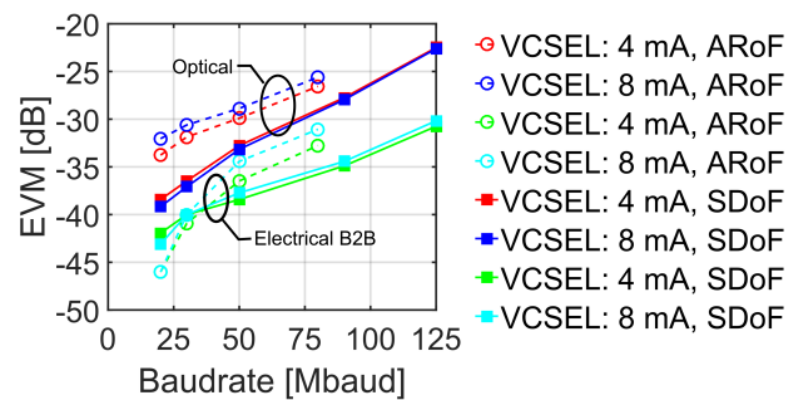

Fig. 12: EVM when the transmitter is directly connected to the VSA (electrical B2B) and EVM when the signal is transmitted over a link of a few meters.

both in-phase and quadrature components, using two baseband sigma-delta modulated signals. This modulated wavelength is combined with a second wavelength, which is spaced the RF carrier frequency apart from the first wavelength. When detecting the two wavelengths with a photodiode, beating occurs and a modulated carrier frequency is provided to the RRH. Fig.13 shows a block diagram of this approach, which may be combined with the system concept depicted in Fig.3 where the modulated wavelength is transmitted over a horizontal fiber and the second wavelength is transmitted over a vertical fiber.

Following this approach, the bandwidth requirements of the optical signal modulation is significantly relaxed as only the baseband signals need to be modulated. This allows to realize much higher baud rates. For example, 100 Gbps electroabsorption modulators have been demonstrated [13], using these with a modest oversampling rate of 16 can provide a signal-to-noise ratio of $39 \mathrm{~dB}$ over a bandwidth of $6.25 \mathrm{GHz}$ using a second order sigma-delta modulator, and thanks to

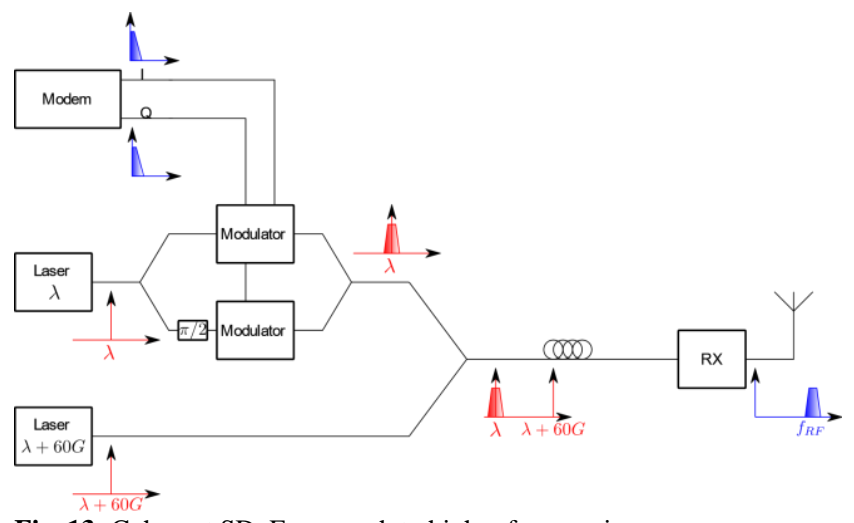

Fig. 13: Coherent SDoF approach to higher frequencies. 
silicon photonics integration [14], the presented architecture is readily implementable.

\section{EXPERIMENTAL ASSESSMENT OF INTERFERENCE AND EXPOSURE}

Constructing a communication system based on a sea of antennas imposes two additional problems. First, the electromagnetic radiation of these antennas should stay within limits to ensure public health. Luckily, an antenna only needs limited radiation power due to the short transmission distance. Nevertheless, the exposure to a human body must be evaluated. Second, to sustain multiple connections simultaneously within a small area, the interference between these transmitters needs to be sufficiently low. To evaluate this, the interference between different cells is measured.

\section{A. Exposure assessment}

The human body is electromagnetically a lossy medium that absorbs radiofrequency (RF) electromagnetic fields (EMFs). In 1998, the international commission on non-ionizing radiation protection (ICNIRP) issued guidelines [15] to protect the public against known adverse health effects of RF EMFs by defining basic restrictions on the whole-body and localized absorption in

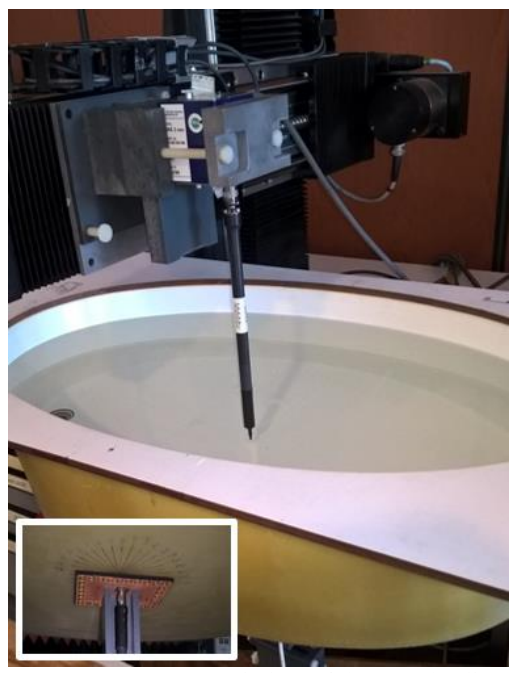

Fig. 14: SAR assessment setup consisting of the standardized flat phantom, dosimetric probe, automated positioning system, and ATTO cell antenna positioned below the flat phantom (inset).

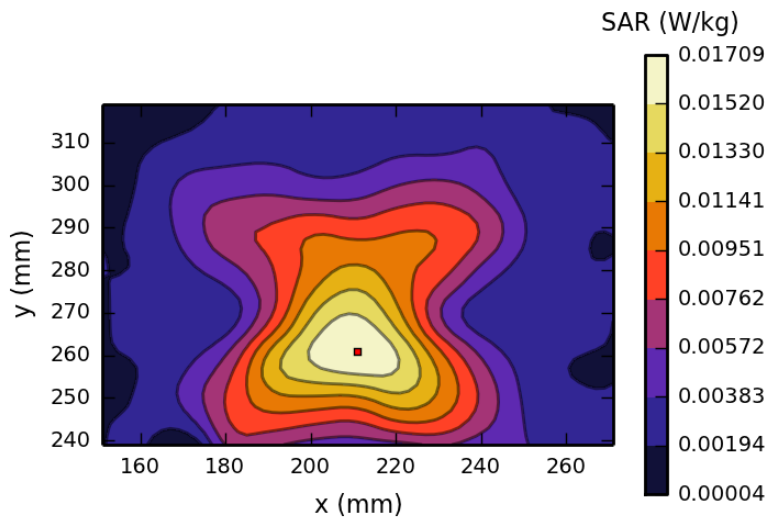

Fig 15: SAR distribution at $5 \mathrm{~mm}$ inside the liquid.

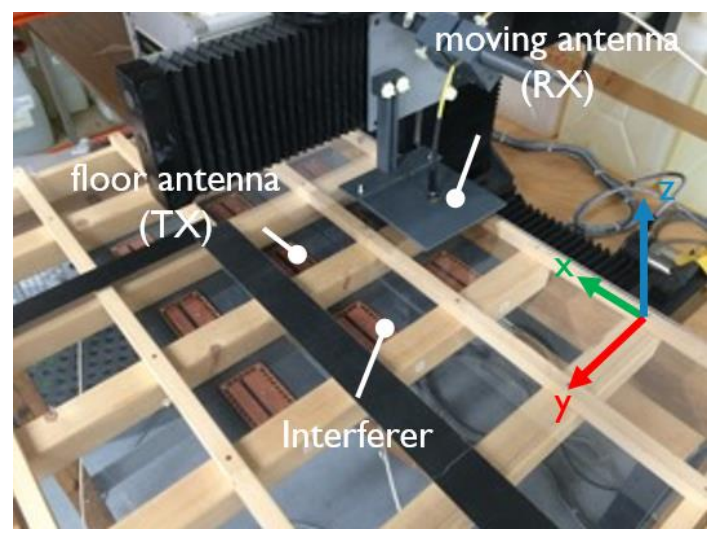

Fig 16: Interference measurement setup consisting a 3x3 ATTO floor cell and the moving antenna attached to the automatic positioning system. Interfering cell and communicating cells are indicated.

terms of Specific Absorption Rate (SAR). The SAR, in W/kg, is the rate at which electromagnetic energy is absorbed in human tissue.

We assessed the compliance of a radiating 3.5 GHz ATTO floor cell with ICNIRP basic restrictions on the localized SAR according to the measurement standard IEC 62209-2 [16]. The measurement setup (see Fig. 14) consisted of the oval flat phantom ELI4 (SPEAG, Zurich, Switzerland) filled with tissue simulating liquid HSL2450 (SPEAG, Zurich, Switzerland), the automatic positioning system Pythron IXE $\alpha-\mathrm{C}-\mathrm{T}$ robot (Phytron GmbH, Gröbenzell, Germany) and the dosimetric probe EX3Dv4 (SPEAG, Zurich, Switzerland). The antenna of the ATTO floor cell was placed below the flat surface of the phantom at $10 \mathrm{~mm}$ from the tissue simulating liquid and radiated continuously $1 \mathrm{~mW}$ at $3.5 \mathrm{GHz}$. These transmitter settings yielded a worst-case exposure scenario. The measured dielectric properties of HSL2450 at $3.5 \mathrm{GHz}$ were: a relative permittivity $\varepsilon_{\mathrm{r}}$ of 36.1 and a conductivity $\sigma$ of $2.47 \mathrm{~S} / \mathrm{m}$. Fig. 15 shows the SAR distribution induced by the ATTO floor antenna $5 \mathrm{~mm}$ inside the liquid. The peak mass-averaged SAR in $10 \mathrm{~g}$ (calculated from the measured SAR distribution) equaled 9.7 $\mathrm{mW} / \mathrm{kg}$ - total expanded uncertainty $(\mathrm{k}=2)$ of the setup is $16 \%$ - This is more than 200 times below the ICNIRP basic restriction of $2 \mathrm{~W} / \mathrm{kg}$ thanks to the low radiating power of the ATTO cell.

\section{B. Interference assessment}

Interference is an important aspect in the design of an ultradense wireless network, especially when the inter-cell distance is in the order of decimeters such as in an ATTO cell. We

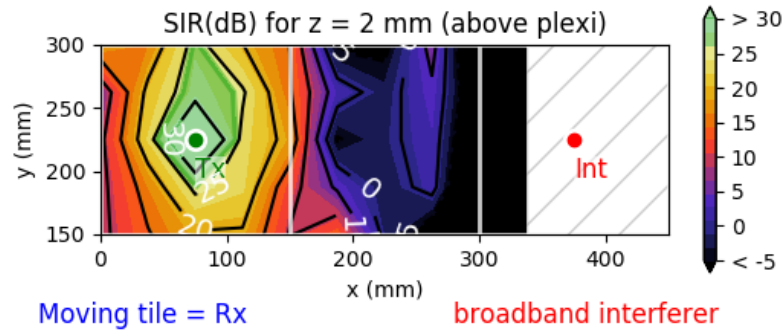

Fig 17: Signal-to-Interference Ratio at the mobile node $(\mathrm{Rx})$ when it moves $2 \mathrm{~mm}$ above the ATTO floor cell. The interfering cell (Int) is located next to the transmitting cell (Tx). The targeted high data-rates per area are achieved for SIR values above $26 \mathrm{~dB}$. The hatched area designates the area outside the range of the automatic positioning system. 
assessed the inference in an ATTO floor cell for the worst-case scenario of a neighboring interfering cell that transmitted with the same power as the communicating cell and all antennas having the same polarization. Fig. 16 depicts the measurement setup consisting of a 3x3 floor cell and a mobile node attached to the automated positioning system Pythron IXE $\alpha-\mathrm{C}-\mathrm{T}$ robot. The mobile node moved stepwise in a plane $2 \mathrm{~mm}$ above the floor. Using the vector network analyzer ZNB $20,100 \mathrm{kHz}-20$ $\mathrm{GHz}$ (Rohde \& Schwarz, Munchen, Germany), we measured the transmission coefficient $\mathrm{S}_{21}$ at $3.5 \mathrm{GHz}$ between the communicating cells and between the interferer and the receiving cell. The interferer is spaced $300 \mathrm{~mm}$ from the transmitting cell. From the measured transmission coefficients, we calculated the signal-to-interference ratio (SIR) - SIR defined as the ratio of $S_{21 \text {,signal }}$ and $S_{21 \text {,interferer }- \text { at the terminals }}$ of the moving antenna (played the role of receiver). Fig. 17 shows the SIR at the mobile node when it moves $2 \mathrm{~mm}$ above the floor. The whole area above the transmitter cell has sufficient SIR to provide a bit error rate below $10^{-5}$ for 16 QAM. The center area above the transmitter even exhibits an SIR larger than $26 \mathrm{~dB}$, which can provide a bit error rate below $10^{-5}$ for 64-QAM. These numbers are obtained with an interferer only $300 \mathrm{~mm}$ from the transmitter, extrapolation based on the path loss, suggest sufficient SIR is available to achieve the targeted high data rates per area in an ATTO cell.

\section{CONCLUSIONS}

This paper illustrated a scalable concept for ultra-high density wireless connectivity. Ultra-small ATTO-cells of only $15 \times 15 \mathrm{~cm}^{2}$ together with mm-wave $3 \times 3$ line-of-sight MIMO allow to reach $100 \mathrm{Gbps}$ wireless throughput. Furthermore, using the path loss and directivity at $60 \mathrm{GHz}$ may be exploited to realize low interfering cells in close vicinity of each other.

To reduce cost and power, most of the ATTO-backend is aggregated in an ATTO-gateway. In this way, overprovisioning of hardware can be limited to the amount of expected users. To ensure sufficient bandwidth and allow easy reconfigurability, a coherent passive optical network carrying the baseband signals is proposed. Information on different wavelengths can be selected from the data fibers by using a separately provided local oscillator wavelength.

Two concepts, which pave the way for a low-cost ATTO cell, have been demonstrated at a lower frequency: a fully passive transmit opto-antenna and low-cost all-digital electro-optical transmitter. Moreover, also the interference between neighboring ATTO cells and the exposure was evaluated to ensure the realization of high throughput per unit area.

\section{REFERENCES}

[1] A. Gupta and R. K. Jha, "A Survey of 5G Network: Architecture and Emerging Technologies", IEEE Access, vol. 3, no. 7, pp. 1206-1232 (2015).

[2] G. Torfs, H. Li, S. Agneessens, J. Bauwelinck, O. Caytan, W. Joseph, H. Rogier, A. Thielens, D. Vande Ginste, X. Yin and P. Demeester, “ATTO: Wireless networking at fiber speed", European Conference on Optical Communication, Sweden, 2017.

[3] L. Verma, M. Fakharzadeh and S. Choi, "Wifi on steroids: 802.11AC and 802.11AD," in IEEE Wireless Communications, vol. 20, no. 6, pp. 30-35, December 2013.

[4] G.P. Fettweis, "The Tactile Internet: Applications and Challenges", IEEE Vehic. Tech. Mag., vol. 9, no. 1, pp. 64-70 (2014).

[5] D. Novak et al., "Radio-Over-Fiber Technologies for Emerging Wireless Systems," in IEEE Journal of Quantum Electronics, vol. 52, no. 1, pp. 111, Jan. 2016.

[6] G. Matthaei, L. Young, and E. Jones, Microwave filters, impedancematching networks, and coupling structures, ser. Artech House Microwave Library.

[7] O. Caytan, S. Lemey, S. Agneessens, and H. Rogier, "SIW antennas as hybrid energy harvesting and power management platforms for the internet of things," International Journal of Microwave and Wireless Technologies, vol. 8, no. 4-5, pp. 767-775, 2016.

[8] S. Lemey, O. Caytan, D. Vande Ginste, P. Demeester, H. Rogier and M. Bozzi, "SIW cavity-backed slot (multi-)antenna systems for the next generation IoT applications," 2016 IEEE Topical Conference on Wireless Sensors and Sensor Networks (WiSNet), Austin, TX, 2016, pp. 75-77."

[9] Q. Van den Brande, S. Lemey, J. Vanfleteren, and H. Rogier, "HighlyEfficient Impulse-Radio Ultra-Wideband Cavity-Backed Slot Antenna in Stacked Air-Filled Substrate-Integrated-Waveguide Technology," IEEE Transactions on Antennas and Propagation, vol. 2017, 9 pages, submitted in 2017.

[10] "Common Public Radio Interface (CPRI)." [Online]. Available: http://www.cpri.info

[11] L. Breyne, G. Torfs, X. Yin, P. Demeester and J. Bauwelinck, "Comparison Between Analog Radio-Over-Fiber and Sigma Delta Modulated Radio-Over-Fiber," in IEEE Photonics Technology Letters, vol. 29, no. 21, pp. 1808-1811, Nov.1, 12017.

[12] L. Bogaert, J. Van Kerrebrouck, A. Abbasi, J. Lambrecht, G. Torfs, X. Yin, G. Roelkens and J. Bauwelinck, "Resonant optical receiver design by series inductive peaking for sub-6 GHz RoF", Microwave and Optical Technology Letters, 59(9), p.2279-2284 (2017)

[13] J. Verbist, M. Verplaetse, A. Srinivasan, P. De Heyn, T. De Keulenaer, R. Vaernewyck, R. Pierco, A. Vyncke, P. Verheyen, S. Balakrishnan, G. Lepage, M. Pantouvaki, P. Absil, X.Yin, G. Roelkens, G. Torfs, J. Van Campenhout and J. Bauwelinck, "Real-Time $100 \mathrm{~Gb} / \mathrm{s}$ NRZ-OOK Transmission with a Silicon Photonics GeSi Electro-Absorption Modulator", IEEE Optical Interconnects Conference (OI) 2017, United States, p.29-30 (2017).

[14] M. Pantouvaki et al., "Active Components for 50 Gb/s NRZ-OOK Optical Interconnects in a Silicon Photonics Platform," in Journal of Lightwave Technology, vol. 35, no. 4, pp. 631-638, Feb.15, 152017.

[15] International Comission on Non-Ionizing Radiation Protection, 1998. Guidelines for limiting exposure to time-varying electric, magnetic, and electromagnetic fields (up to $300 \mathrm{GHz}$ ). Health physics, 74(4), pp.494522.

[16] International Electrotechnical Commission, 2010. IEC 62209-2 ed. 1: Human exposure to radio frequency fields from hand-held and bodymounted wireless communication devices - Human models, instrumentation, and procedures - Part 2: Procedure to determine the specific absorption rate (SAR) for wireless communication 